\title{
Highly Compressed Ion Beams for High Energy Density Science
}

A. Friedman, J. J. Barnard, R. J. Briggs, D. A. Callahan, G. J.

Caporaso, C. M. Celata, R. C. Davidson, A. Faltens, L. Grisham, D. P. Grote, E. Henestroza, I. Kaganovich, E. P. Lee, R. W. Lee, M. Leitner, B. G. Logan, S. D. Nelson, C. L. Olson, G. Penn, L. R. Reginato, T. Renk, D. Rose, A. Sessler, J. W. Staples, M. Tabak, C. Thoma, W. Waldron, D. R. Welch, J. Wurtele, S. S. Yu

May 12, 2005

Particle Accelerator Conference 2005

Knoxville, TN, United States

May 16, 2005 through May 20, 2005 
This document was prepared as an account of work sponsored by an agency of the United States Government. Neither the United States Government nor the University of California nor any of their employees, makes any warranty, express or implied, or assumes any legal liability or responsibility for the accuracy, completeness, or usefulness of any information, apparatus, product, or process disclosed, or represents that its use would not infringe privately owned rights. Reference herein to any specific commercial product, process, or service by trade name, trademark, manufacturer, or otherwise, does not necessarily constitute or imply its endorsement, recommendation, or favoring by the United States Government or the University of California. The views and opinions of authors expressed herein do not necessarily state or reflect those of the United States Government or the University of California, and shall not be used for advertising or product endorsement purposes. 


\title{
HIGHLY COMPRESSED ION BEAMS FOR HIGH ENERGY DENSITY SCIENCE*
}

\author{
A. Friedman ${ }^{\dagger 1,2}$, J.J.Barnard ${ }^{1,2}$, R.J.Briggs ${ }^{7}$, D.A.Callahan ${ }^{2}$, G.J.Caporaso ${ }^{2}$, C. M.Celata ${ }^{1,3}$. $^{1}$. \\ R.C.Davidson $^{1,4}$, A.Faltens ${ }^{1,3}$, L.Grisham ${ }^{1,4}$, D.P.Grote ${ }^{1,2}$, E.Henestroza ${ }^{1,3}$, I.Kaganovich $^{1,3}$, \\ E.P.Lee ${ }^{1,3}$, R.W.Lee ${ }^{2}$, M.Leitner ${ }^{1,3}$, B. G.Logan ${ }^{1,3}$, S.D.Nelson ${ }^{2}$, C. L.Olson ${ }^{1,5}$, G. Penn ${ }^{3}$,

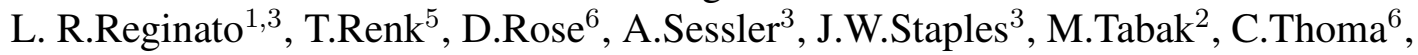 \\ W.Waldron ${ }^{1,3}$, D.R.Welch ${ }^{6}$, J.Wurtele ${ }^{3}$, and S.S.Yu ${ }^{1,3}$ \\ ${ }^{1}$ Heavy Ion Fusion Virtual National Laboratory; ${ }^{2}$ LLNL, Livermore, CA; ${ }^{3}$ LBNL, Berkeley, CA; \\ ${ }^{4}$ PPPL, Princeton NJ; ${ }^{5} \mathrm{SNL}$, Albuquerque NM; \\ ${ }^{6}$ ATK Mission Research Corp., Albuquerque NM; ${ }^{7}$ SAIC, Alamo CA
}

\begin{abstract}
The Heavy Ion Fusion Virtual National Laboratory (HIFVNL) is developing the intense ion beams needed to drive matter to the High Energy Density (HED) regimes required for Inertial Fusion Energy (IFE) and other applications. An interim goal is a facility for Warm Dense Matter (WDM) studies, wherein a target is heated volumetrically without being shocked, so that well-defined states of matter at 1 to $10 \mathrm{eV}$ are generated within a diagnosable region. In the approach we are pursuing, low to medium mass ions with energies just above the Bragg peak are directed onto thin target "foils," which may in fact be foams with mean densities $1 \%$ to $10 \%$ of solid. This approach complements that being pursued at GSI, wherein high-energy ion beams deposit a small fraction of their energy in a cylindrical target. We present the beam requirements for WDM experiments. We discuss neutralized drift compression and final focus experiments and modeling. We describe suitable accelerator architectures based on Drift-Tube Linac, RF, single-gap, Ionization-Front Accelerator, and Pulse-Line Ion Accelerator concepts. The last of these is being pursued experimentally in the HIF-VNL. Finally, we discuss plans toward a user facility for target experiments.
\end{abstract}

\section{ION-BEAM HEATING OF MATTER}

Recently, much interest has developed in the emerging field of High Energy Density Physics (HEDP) [1, 2, 3]. Because the ultimate interest of the Heavy Ion Fusion program is to heat an inertial fusion target to conditions of high energy density, and because the intense ion beams used by the program offer significant advantages in the controlled heating of matter, the pursuit of HEDP experiments has become one near-term focus of the program. The goal is to produce a heavy ion driver for a HEDP experimental facility within the next decade. The innovation necessary for such a facility is driving major changes in the program, as

\footnotetext{
* Work performed under auspices of USDOE by the University of California Lawrence Livermore and Lawrence Berkeley National Laboratories, the Princeton Plasma Physics Laboratory, and Sandia National Laboratory, under Contract Nos. W-7405-Eng-48, DE-AC03-76SF00098, DEAC02-76CH03073, and DE-AC04-94AL85000, and by ATK Mission Research Corp. and Science Applications International Corp.

$\dagger$ af@1lnl.gov
}

described herein. In particular the means to deliver the required energy into a small (mm-scale) spot in a time shorter than the hydrodynamic expansion time of the target matter, typically a nanosecond, is being addressed.

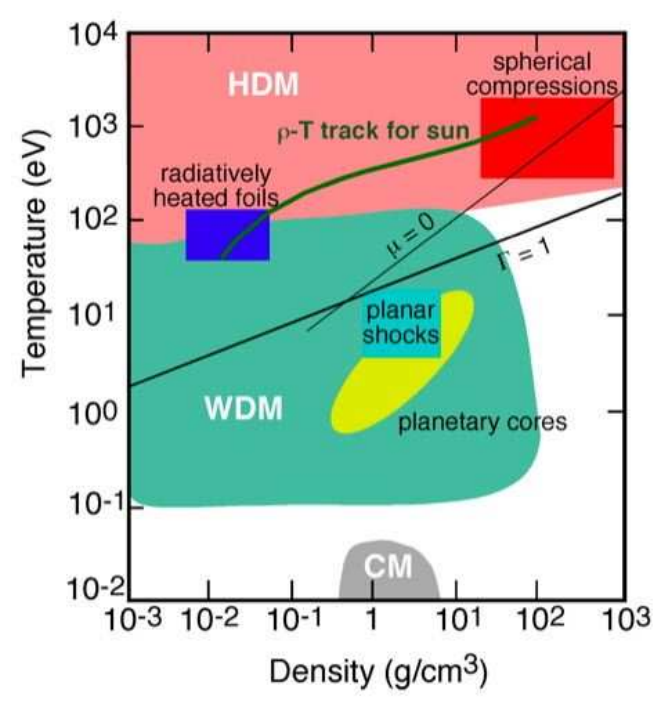

Figure 1: Hydrogen phase diagram indicating the high energy density regime separated into the hot dense region (pink) and the warm dense region (green).

In Fig. 1 the Hydrogen phase diagram is depicted. Various states found in nature are indicated on the graph. Below the line $\mathrm{G}=1$, strong coupling effects are important. The condensed matter phase is indicated by the gray region. Dense, strongly coupled plasmas in the Warm Dense Matter regime at temperatures of 0.1 to $10 \mathrm{eV}$ and densities $10^{-2}$ to $10^{-1} \mathrm{~g} / \mathrm{cm}^{2}$ are productive areas to test EquationOf-State (EOS) models. Indeed, it is in this region where the discrepancies in EOS models can be $20-40 \%$ or more, e.g., in the pressure predicted for Aluminum by two commonly used models. The reader is referred to the overview by R. Lee in [4], from which Fig. 1 was taken. The regime is interesting and theoretically challenging because material under these conditions is neither a classical plasma nor ordinary condensed matter. For these reasons, and because the regime will be accessible to ion drivers with modest effort, experiments in this regime will be undertaken first. 
Ion beam heating offers unique opportunities for High Energy Density Science. Two general approaches to such heating have been identified. In the first, being pursued at GSI in Germany[5, 6], long $(\sim 3 \mathrm{~mm})$ cylindrical targets are heated by energetic beams, e.g. of order a kiloJoule of $40-\mathrm{GeV}$ Uranium, that deposit a small fraction of their energy in the targets, heating them to temperatures of order an eV. Such an approach makes good use of the existing and planned facilities at GSI. In contrast, the U.S. Heavy Ion Fusion Virtual National Laboratory is planning to heat thin foils using low- to medium-mass ions of much lower kinetic energy, e.g. $\sim 2.4 \mathrm{~J}$ of $24 \mathrm{MeV} \mathrm{Na}^{+}$. The beam ions slow down through the Bragg peak energy as they pass through the foil, so as to maximize the deposition rate $d E / d x$ and uniformity, heating a $1-\mathrm{mm}$ radius volume of the target to electron temperatures of a few $\mathrm{eV}$ in $\sim 1 \mathrm{~ns}$.

This latter approach is expected to have a number of attractive attributes, incuding: volumetric energy deposition involving no shocks and without either x-ray or electron preheating of the sample; large volumes, uniformly heated to within a few percent, facilitating diagnostics; time scales long enough for equilibrium conditions to prevail; deposition of perhaps 3/4 of the beam energy in the sample, allowing an accurate accounting of the beam energy loss as a measure of the energy deposited; and a high repetition rate, valuable for experimental setup and diagnostic tuning.

A workshop in October, 2004 brought together experts in targets, HEDP/WDM physics, and accelerators. Much of this material was drawn from that workshop and followon meetings; the Proceedings are available online [4].

\section{BEAM REQUIREMENTS \& APPROACH}

The requirements for heating a target to $\sim 3 \mathrm{eV}$ for measurements of WDM properties have been assessed using analysis and a state-of-the-art hydrodynamics code, Hydra [7]. A possible parameter set is listed in Table 1.

Table 1: Example beam requirements for $\sim 3 \mathrm{eV}$ regime.

\begin{tabular}{|c|c||c|c|}
\hline Ion & $\mathrm{Na}^{+}$ & Atomic no. & 23 \\
$\mathrm{Q}_{\text {total }}$ & $0.1 \mu \mathrm{C}$ & No. ions & $610^{11}$ \\
$\epsilon_{\mathrm{nx}}$ & $2.3 \mathrm{~mm}-\mathrm{mrad}$ & Focal length & $70 \mathrm{~cm}$ \\
$\epsilon_{\mathrm{nz}}$ & $33 \mathrm{~mm}-\mathrm{mrad}$ & Focal solenoid & $15 \mathrm{~T}$ \\
$\mathrm{r}_{\text {spot }}$ & $1 \mathrm{~mm}$ & & \\
\hline \hline & $\mathrm{At}$ & Before & At final focus \\
& injector & compression & (neutralized) \\
\hline Energy & $1.0 \mathrm{MV}$ & $23.5 \mathrm{MV}$ & $(\beta=0.047)$ \\
Duration & $177 \mathrm{~ns}$ & $20 \mathrm{~ns}$ & $1 \mathrm{~ns}$ \\
Length & $0.5 \mathrm{~m}$ & $0.28 \mathrm{~m}$ & $0.014 \mathrm{~m}$ \\
Perveance & $1.810^{-3}$ & $1.410^{-4}$ & $2.810^{-3}$ \\
$\delta p / p$ & $210^{-3}$ & $710^{-4}$ & 0.015 \\
\hline
\end{tabular}

A much-revised program based on innovative methods is being pursued to meet these requirements. New approaches have been adopted in five areas: beam production, acceleration, transport (transverse confinement), temporal compression, and focusing onto the target.
The beam entering the accelerator is, ideally, shorter in duration, with a higher line-charge density, than was previously assumed. Several injector approaches are possible, but the one that has received the most attention is "acceldecel / load-and-fire" [8]. In this approach, a high current is achieved (via the Child-Langmuir scaling) by use of a high voltage across the initial diode gap, but then the beam is immediately decelerated across a second gap. Because the current is constant (at mid-pulse), the line charge density increases. This dense beam is "loaded" into a solenoid and then "fired" (accelerated all at once), perhaps by a resistively graded line. Other scenarios are possible. A shortgap diode can also generate a high line-charge density at low voltage, perhaps with magnetic insulation. Also, a Pulse-Line Ion Accelerator (discussed below) operating in "snowplow" mode may replace the resistively graded line.

The induction accelerators that has been pursued in the U.S., while efficient and attractive for IFE, do not match well to short pulses, and have small accelerating gradients at low energy. Furthermore, the fact that a large total amount of energy is not needed for HEDP/WDM purposes opens the door to other approaches which are well suited for accelerating short pulses, but which may not extrapolate to the longer pulses needed for IFE. The approaches studied are discussed in the second section following.

Transport using solenoid focusing scales well to high line charge densities and low kinetic energies; in contrast, multi-beam quadrupole arrays have been the baseline approach for the IFE mission. Solenoid transport experiments are planned for the near future.

Temporal compression is best carried out using neutralized drift compression (that is, imparting a velocity gradient or "tilt" from head to tail on the beam, and allowing it to drift); this contrasts with the un-neutralized drift compression usually considered for the IFE mission. Transverse space charge forces must be minimized while the beam passes through the target chamber and onto the target, and so in this region the beam must be neutralized (for both IFE and HEDP applications). Neutralized chamber transport and neutralized drift compression experiments and modeling are discussed in the following section.

An advantage of the un-neutralized drift compression previously assumed is that the space charge causes the inward flow (in the beam frame) to stagnate, removing most of the coherent velocity variation along the beam and easing the chromatic acceptance requirements of the final focusing system. In contrast, un-neutralized drift compression leaves the beam with a large velocity spread at final focus, which must be accommodated. Possible approaches include use of a strong solenoid, a plasma lens, and/or a plasma channel pinch, alone or in combination. A graded solenoid may be attractive, in that it can provide adiabatic matching from the upstream line. Upstream corrections using pulsed lenses are also possible. See the articles by E. P. Lee, Y-J. Chen, and S. S. Yu in [4].

For HEDP/WDM applications, the accelerator, drift compression, and final focus must all work together. In one 
concept, the beam is accelerated while being confined in Brillouin flow by a series of solenoids; given a 5-10\% velocity tilt; neutralized as it enters a Neutralized Drift Compression (NDC) region with a weak (kG-level) guide field; is focused (while still in plasma) by a strong solenoid; and finally focused more tightly onto the target by an assistedpinch discharge channel. Key issues include the effectiveness of the dipole "trap" (upstream of the NDC region) at preventing plasma flow upstream; the transition from Brillouin flow to neutralized transport; and control of beam plasma instabilities and stripping in the long plasma column. See the article by Welch in [4].

\section{NEUTRALIZED TRANSPORT, FOCUSING, \& COMPRESSION STUDIES}

The Neutralized Transport Experiment (NTX) was used to study the behavior of a converging ion beam in a target chamber. The apparatus, with the flexibility to introduce plasmas of varying density from two different sources along the beam path, is shown in Fig. 2. NTX quantified the

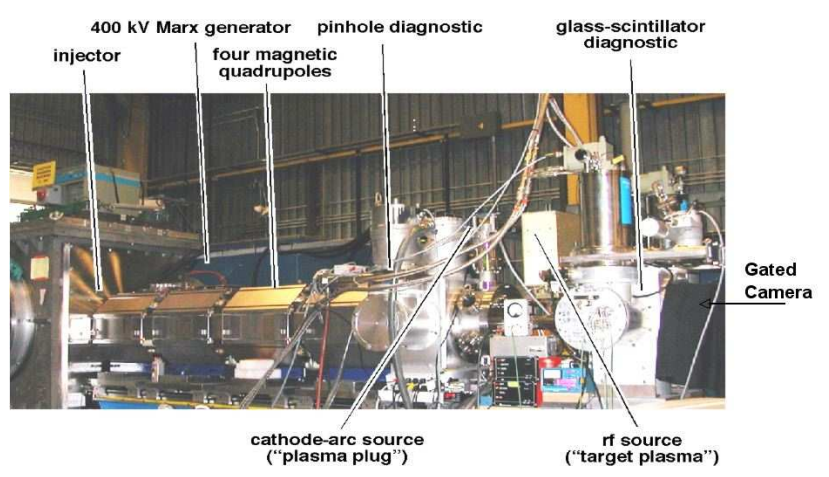

Figure 2: NTX experiment at LBNL.

improvement in focus when plasma was present; an example (with a $6 \mathrm{~mA}$ beam) is shown in Fig. 3. More detailed studies, including LSP simulations, were carried out [9].
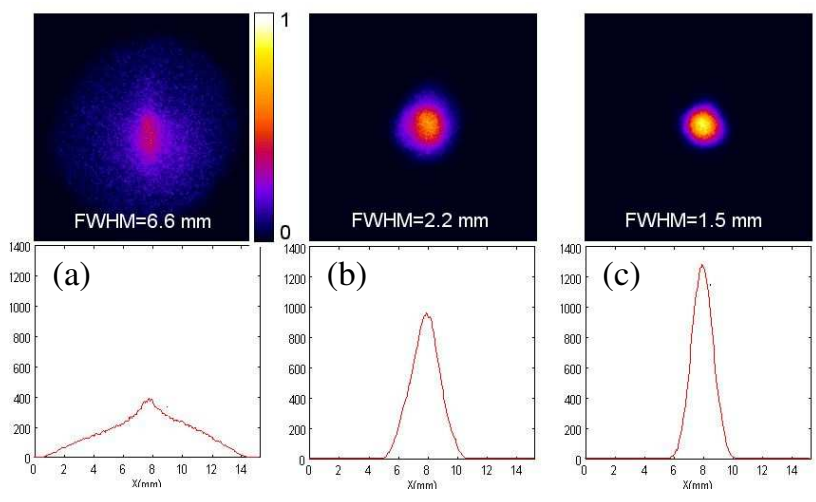

Figure 3: Images at focal plane of NTX: (a) un-neutralized; (b) plasma plug; (c) plasma plug and volume plasma.

A revision of the NTX apparatus, the Neutralized Drift Compression Experiment (NDCX-1a), uses an induction

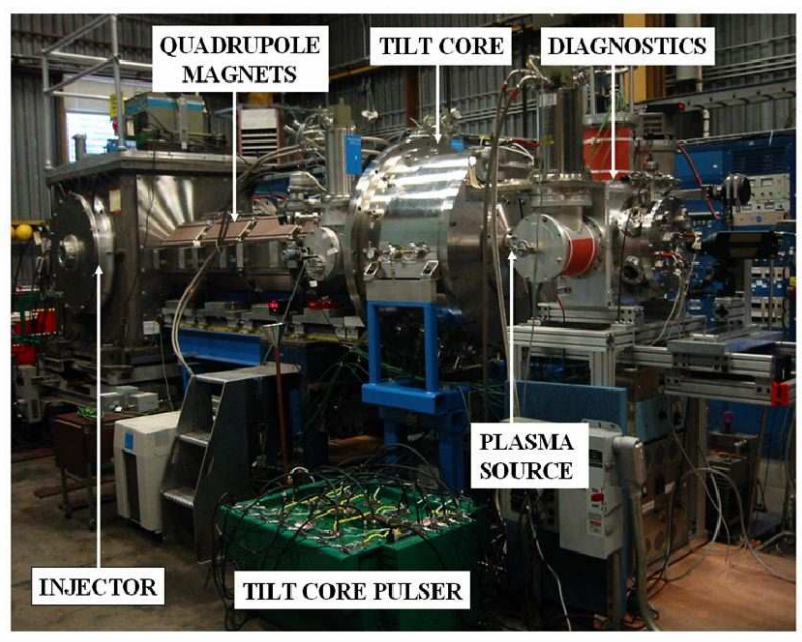

Figure 4: NDCX experiment at LBNL.

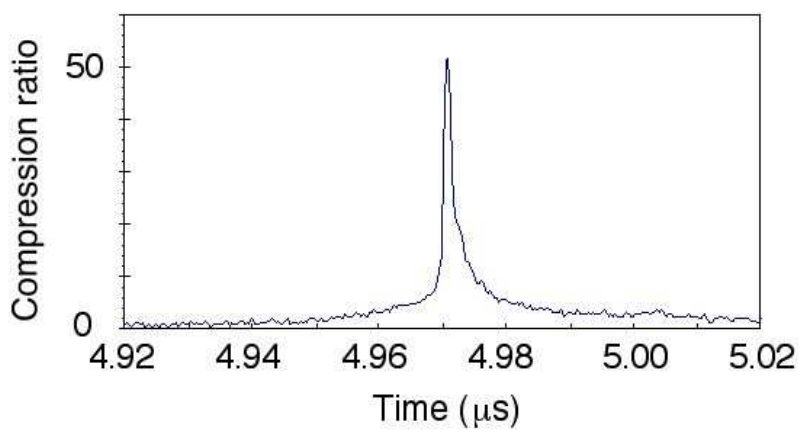

Figure 5: Compression ratio vs. time in NDCX, as measured using a phototube.

core to impart a velocity "tilt" to a section of the $25 \mathrm{~mA}$, $255 \mathrm{keV}$ beam. The layout is shown in Fig. 4. Compression factors of order fifty were recently measured, using a phototube $[10,11,12,13]$. See Fig. 5. LSP simulations support these experiments $[14,15,16]$. Simulations and analysis are also developing our general understanding of beam flows in plasmas $[17,18]$.

\section{ACCELERATOR CONCEPTS}

The Workshop in October, 2004 [4] identified five approaches to ion acceleration in an HEDP/WDM research facility. Four of these are discussed briefly, then the PulseLine Ion Accelerator (PLIA), which is the approach currently being pursued, is described in slightly greater detail.

The pulsed Drift-Tube Linac (DTL) was, in the early days of the U.S. HIF program, thought of as an extended injector for an induction linac with magnetic quadrupoles; a DTL was the first major HIF experiment at LBNL. For the HEDP/WDM application, it is especially well matched, since the short pulses allow drift tubes of modest length. At the Workshop, and during follow-on research, both a design based on existing experience and an "optimistic" 
variation were developed. The first accelerates 16 parallel $\mathrm{K}^{+}$beams, each with line charge density of $0.25 \mu \mathrm{C} / \mathrm{m}$, to $10 \mathrm{MeV}$ over about $10 \mathrm{~m}$; the second uses a stripper and charge selector at around $4 \mathrm{MeV}$ before acceleration to 40 $\mathrm{MeV}$. See the article by Faltens in [4].

Radio-frequency (RF) linacs have not traditionally been used to accelerate ions at kA-level currents to modest energies. The GSI group is using RF acceleration, but with heavy ions stripped to high charge levels for experiments at energies well above the Bragg peak, so ion space charge is not a major difficulty. For the present application, a number of scenarios were explored. The most promising employed multiple parallel beams to transport, and accelerate, the necessary current. The many beams (about 16) are combined only as they begin to impinge on the target. Solenoid transport appears to be attractive, but inter-beam effects have to be minimized. A number of necessary further studies were identified. See [19] and the articles by Staples, Keller, Ostroumov, and Chou in [4].

A single-gap accelerator based on pulsed-power technology also appears capable of meeting the requirements for HEDP/WDM studies in the regime being considered. Indeed, pulsed diodes have already generated conditions close to $100 \mathrm{eV}$. However, the shortest pulses in those machines have been 10-20 ns, not $1 \mathrm{~ns}$. The transverse temperatures have been large, implying that the beam must be drift-compressed at a relatively large radius, and then focused onto the target via a short focal-length cell. For the HEDP application, in contrast with the inertial fusion mission, efficiency is not a requirement, and a simple bipolar diode with a high-impedance driver may offer the possibility of better beam quality than can be obtained in a diode which magnetically suppresses electron backflow. See the article by Olson, Ottinger, and Renk in [4].

The Ionization-Front Accelerator (IFA) uses an intense relativistic electron beam (IREB) in conjunction with a laser, the beam of which is swept so that it illuminates successive points along the accelerator axis. The space-charge field at the head of the IREB accelerates the ion bunch; laser ionization of the working gas controls the distance to which the IREB can propagate, so that the accelerating field travels synchronously with the ion bunch. The approach was first verified experimentally in the 1970's, but in that era the required technology was complex. The development of high-power short-pulse laser technology, as well as improvements in pulsed power technology over the last 20 years, motivate further study of the concept, and indeed new research in this area is underway. See [20] and the article by Olson, Ottinger, and Renk in [4].

The Pulse Line Ion Accelerator (PLIA) [21, 22] is based on a helical distributed transmission line that acts as a broad-band traveling-wave accelerator. When driven by a smooth waveform, it operates in a long-wavelength, nondispersive regime, and allows voltage pulses to travel along the beam undistorted. A rising pulse applied to the upstream end appears at any instant in time as a spatial voltage ramp; there is a traveling region containing an acceler- ating field along with which an ion pulse can travel and be steadily accelerated. This affords increases in ion energy that can greatly exceed the applied voltage. The system is inexpensive; it favors short pulses because they correspond to higher accelerating fields for any given voltage-holding limit. Further voltage multiplication can be obtained by coupling in the drive through a primary winding wrapped around one end of the helix [21, 23]. See the article by Friedman in [4].

A lumped-element circuit model can describe wave behavior on the helix; elements include the capacitance of the helix to ground, the inter-turn capacitances, and the self- and mutual inductances of the turns. At the long wavelengths of interest, there is very little Bessel function falloff of the accelerating field toward the axis. Using such a model, the two principal operating modes can be illustrated; these are the short-pulse ("surfing") mode appropriate to further acceleration of an established beam, and the long pulse ("snowplow") mode suitable for launching a slow-moving beam in a scenario resembling "load-andfire." See Fig. 6 for an illustration of these modes.

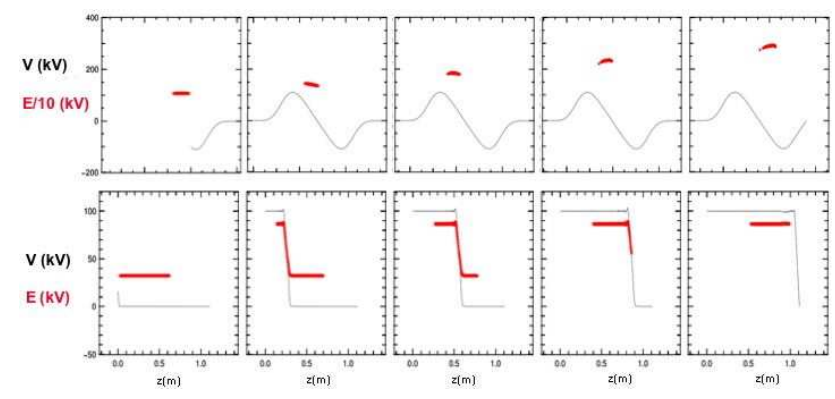

Figure 6: Model of helix operation. Upper panel: short beam "surfs" on traveling voltage pulse (snapshots in wave frame); lower panel: longer beam is accelerated by snowplow (snapshots in lab frame).

More sophisticated simulation studies of PLIA behavior are being carried out. WARP code simulations (using a lumped circuit model to describe the helix) are clarifying beam dynamics in the helix under the influence of space charge and circuit loading, and play a key role in experiment design. See [8, 24]. Electromagnetic modeling is also underway [25].

As of this writing, experiments using a test PLIA module to accelerate a section of the NDCX beam are beginning. The module is shown in Figs. 7 and 8. Beam transport through the module has been tested, and acceleration tests are getting underway.

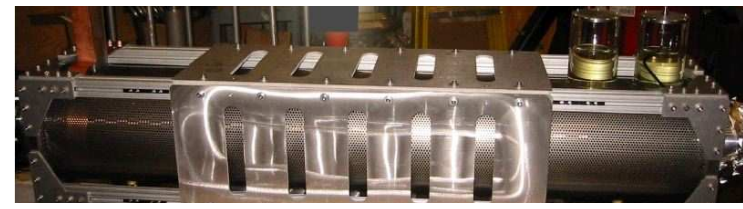

Figure 7: PLIA module before installation on NDCX. 


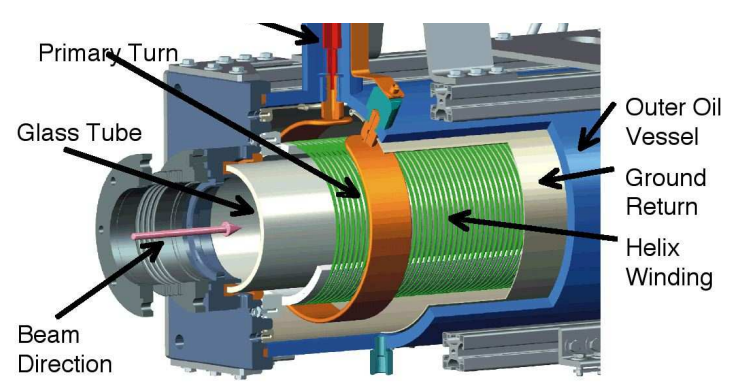

Figure 8: CAD rendering of input end of PLIA module.

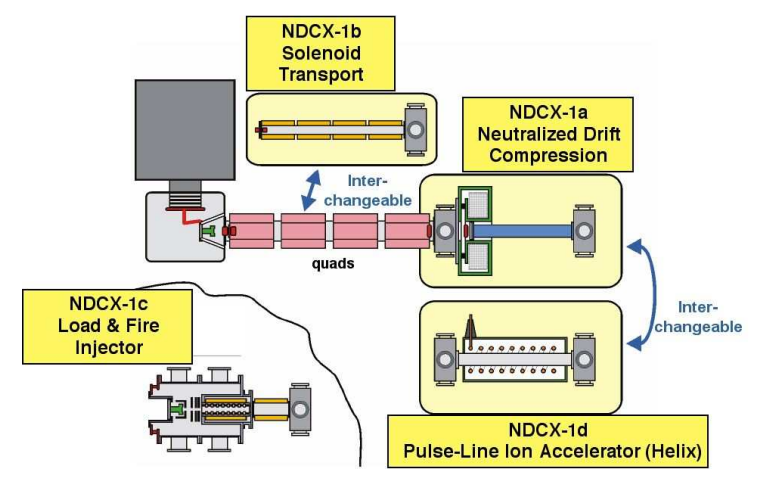

Figure 9: Overview of planned NDCX-1 experiments.

\section{PLANS}

The ongoing and planned experiments center on one facility with inter-changeable parts, to be used for several experiments which will carried out in an interleaved manner. See Fig. 9. NDCX-1a denotes studies of neutralized drift compression; NDCX-1b solenoid transport; NDCX1c high-current injection; and NDCX-1d Pulse-Line acceleration. NDCX-1a, b, and d, to be carried out over the next 2-3 years, can be done with existing equipment; NDCX-1c will require a modest amount of new hardware. By 2009, it is planned that these elements will have been explored, with a PLIA accelerating $0.1 \mu \mathrm{C}$ of $\mathrm{Na}^{+}$to $4 \mathrm{MeV}$. The next step, NDCX-2, will be an integrated experiment at $24 \mathrm{MeV}$, heating targets to a few eV, in the 2010-11 time frame. Then, by adding the necessary target-handling and diagnostic capabilities, over the next few years the NDCX2 will become the core of a rep-rated $(>10 \mathrm{~Hz})$ user facility.

\section{REFERENCES}

[1] "Frontiers in High Energy Density Physics - The X-Games of Contemporary Science," (Nat. Academies Press, 2003).

[2] "Connecting Quarks with the Cosmos:Eleven Science Questions for The New Century (Nat. Academies Press, 2003)";

[3] "Frontiers For Discovery In High Energy Density Physics," (prepared for Nat. Science and Technology Council, 2004);

[4] Proc. Workshop on Accel. Driven High Energy Density Physics, LBNL, Oct. 26-9, 2004, J. J. Barnard, Ed.; on web: http://hifweb.lbl.gov/public/hedpworkshop/toc.html

[5] D. H. H. Hoffmann, et al., Phys. Plasmas 9, 3651 (2002).
[6] N. A. Tahir, et al., "Intense heavy ion beams as a tool to induce high-energy-density states in matter," Contrib. Plasma Phys. 43, 373 (2003).

[7] J. J. Barnard, et al., "Accelerator and Ion Beam Tradeoffs for Studies of Warm Dense Matter," these Proc: RPAP039.

[8] E. Henestroza, et al., "Extraction and Compression of High Line Charge Density Ion Beams," these Proc: FPAT028.

[9] P. K. Roy, et al., "Results on intense beam focusing and neutralization from the neutralized beam experiment," Phys. Plasmas 11, 2890 (2004).

[10] P. K. Roy, et al., "Initial Results on Neutralized Drift Compression Experiments (NDCX) for High Intensity Ion Beam," these Proc: FPAE071.

[11] P. Efthimion, et al., "Ferroelectric Plasma Source for Heavy Ion Beam Charge Neutralization," these Proc: TPAT036.

[12] S. Eylon, et al., "Development of Diagnostics for the Neutralized Ion Beam Drift Compression Experiments (NDCX)," these Proc: RPAT023.

[13] F. M. Bieniosek, et al., "Optical Faraday Cup for Heavy Ion Beams," these Proc: RPAT022.

[14] C. H. Thoma, et al., "LSP Simulations of the Neutralized Drift Compression Experiment," these Proc: FPAE077.

[15] A. Sefkow, "LSP Numerical Simulations of the Neutralized Drift Compression Experiment," these Proc: TPAT068.

[16] D. Rose, "Time-of-Flight Plasma-Neutralized Drift Compression of Heavy Ion Beams for High Energy Density Physics," these Proc: FPAP032.

[17] R. C. Davidson, et al., "Multispecies Weibel and TwoStream Instabilities for Intense Ion Beam Propagation Through Background Plasma," these Proc: FPAP026.

[18] I. Kaganovich, et al., "Ion Beam Pulse Interaction with Background Plasma in a Solenoidal Magnetic Field," these Proc: FPAP028.

[19] J. W. Staples, et al., "RF-Based Accelerators for HEDP Research," these Proc: RPAP023.

[20] D. R. Welch, et al., "Simulation of the Collective Acceleration of 100 Picosecond Ion Pulses with the Ionization Front Accelerator," these Proc: TPAE062.

[21] R. J. Briggs, et al., "Helical Pulseline Structures for Ion Acceleration," these Proc: ROAB005.

[22] G. Caporaso, et al., "Dispersion Analysis of the Pulseline Accelerator," these Proc: FPAT034.

[23] W. Waldron, et al., "High Voltage Operation of Helical Pulseline Structures for Ion Acceleration," these Proc: FPAT029.

[24] D. P. Grote, et al., "Particle Simulations of Acceleration Schemes for High-Current, Short-Bunch, Ion Beams," these Proc: FPAT027.

[25] S. D. Nelson, et al., "Electromagnetic Simulations of Helical-based Ion Acceleration Structures," these Proc: FPAT037. 